\title{
Usages des TIC et rapports a l'incertitude en situation de catastrophes naturelles
}

\section{Claire Brossaud}

\section{(2) OpenEdition Journals}

\section{Édition électronique}

URL : http://journals.openedition.org/developpementdurable/6772

DOI : 10.4000/developpementdurable.6772

ISSN : 1772-9971

\section{Éditeur}

Association DD\&T

\section{Référence électronique}

Claire Brossaud, « Usages des TIC et rapports a l'incertitude en situation de catastrophes naturelles ». Développement durable et territoires [En ligne], Dossier 11 | 2008, mis en ligne le 06 novembre 2008, consulté le 19 avril 2019. URL : http://journals.openedition.org/developpementdurable/6772 ; DOI : 10.4000/developpementdurable.6772

Ce document a été généré automatiquement le 19 avril 2019.

\section{(c) (i) \&)}

Développement Durable et Territoires est mis à disposition selon les termes de la licence Creative Commons Attribution - Pas d'Utilisation Commerciale 4.0 International. 


\title{
Usages des TIC et rapports a l'incertitude en situation de catastrophes naturelles
}

\author{
Claire Brossaud
}

Tempête Limoges 1999. Après le passage d'un premier coup de vent sur le nord de la France dans la nuit du 24 au 25 décembre 1999, l'ouest septentrional de la France est touché. Les rafales soufflent à plus de $200 \mathrm{~km} /$ heure sur l'Ile de Ré et à $173 \mathrm{~km} /$ heure en Ile-de-France. L'après midi et la nuit du 27 décembre, une deuxième tempête balaye le sud de la France. Les vents sont d'une extrême violence, particulièrement sur la partie sud-ouest du pays : $198 \mathrm{~km} /$ heure sur l'île d'Oléron, $148 \mathrm{~km} /$ heure à Limoges. Le Limousin fait partie des régions les plus sinistrées en nombre d'arbres couchés, nombre de personnes isolées, blessées ou décédées. 88 morts au total sont enregistrés les dimanche 26 et lundi 27 décembre en France.

Inondations Abbeville 2001: Les inondations de la Somme ont eu des effets particulièrement dévastateurs, tant par l'importance des crues que par leur persistance dans le temps. Une chronologie des événements peut être établie entre le 18 mars et le 28 juin 2001, période caractérisée par une forte montée des eaux du fleuve due à de très importantes précipitations. L'amplitude de cette crue est particulièrement puissante dans la commune d'Abbeville, où 530 familles doivent être relogées. Cet événement fait par ailleurs l'objet d'une grande médiatisation au niveau national ainsi que d'importantes controverses et rumeurs.

Intempéries Bourg-en-Bresse 2005 : Les 15, 16 et 17 avril 2005, pluies et neige en abondance touchent fortement l'est de la France, le long d'une ligne allant des Vosges à la Haute-Loire : routes coupées, hébergements d'urgence d'automobiliste, 50000 foyers privés d'électricité en Rhône-Alpes et Franche Comté. Des chutes de neige tombent en grande quantité sur les reliefs de l'Ain. Une pluviométrie s'accumule au cours du week-end sur le secteur de Bourg-en-Bresse, faisant suite à une période particulièrement humide. Le dimanche 16 avril au matin, le plan rouge et le plan blanc sont lancés à la Préfecture, afin d'évacuer notamment 84 personnes d'une maison de retraite située dans le quartier des Baudières. Au total, trente familles d'un immeuble sont relogées. 38 propriétés sont touchées directement par les inondations. La décrue intervient dès le 17 avril. 
1 Les technologies de l'information et de la communication (TIC) sont désormais inséparables du développement durable. Elles mesurent des indices de pollution, tracent des produits alimentaires, fluidifient la circulation des transports, rassemblent des ONG environnementales, anticipent des menaces sanitaires et écologiques, etc. Le recours aux outils numériques est aujourd'hui devenu un enjeu socio-politique majeur dans des situations de gouvernance fortement marquées par l'incertitude. Plus la contingence est grande, plus les TIC, et au-delà, les sciences et techniques dans leur ensemble, sont convoquées en effet au chevet d'une société du risque (Beck, 2001 ; Latour, 1999) où il s'agit de protéger l'existant afin de préserver les générations futures. Ainsi, face à l'aléatoire, il est devenu aujourd'hui banal de faire appel à des experts (environnementalistes, psychologues, psycho-sociologues, consultants) et à des technologies innovantes pour intervenir sur toutes sortes de facteurs de dérégulation: identitaires, organisationnels, institutionnels, etc. Les catastrophes naturelles - avalanches, feux de forêt, inondations, mouvements de terrain, cyclones, tempêtes et autres séismes ou éruptions volcaniques - représentent le point d'orgue de ce phénomène. Car malgré les prévisions météorologiques les plus précises, il reste encore impossible d'arrêter un cyclone alors que l'on peut toujours stopper une chaîne de production dans le cas d'un risque industriel. Le statut de l'événement isolé renforce cette absence supposée de contrôle. Et dans l'imaginaire collectif, ces événements incombent généralement le moins à la responsabilité humaine, mais à la force de la nature.

2 En réalité, nombre de travaux ont montré que les représentations des risques naturels et de l'incertitude qui s'y rattachent, pouvaient être socialement construites, notamment par des systèmes d'action fortement territorialisés (Gilbert, 2003) et par la place des controverses techniques dans ce contexte (Lascoume, 2001). Mais peu d'analyses se sont penchées sur le rôle des TIC dans la production d'une histoire commune du risque à des échelles localisées. Nous avons pris cette direction dans le cadre d'une recherche menée par une équipe pluridisciplinaire pour le compte du Ministère de l'Environnement et du Développement Durable (Brossaud, 2005). Celle-ci a permis d'examiner comment des professionnels et des sinistrés qui ont été confrontés à trois catastrophes naturelles, la tempête de décembre 1999 à Limoges, les inondations d'Abbeville en 2001 et des intempéries à Bourg-en-Bresse en avril 2005 se sont emparés des outils numériques (Internet, téléphone portable, bases de données, outils de travail partagés, etc.) avant, pendant et après les événements. Il s'agissait de comprendre plus précisément comment s'effectuaient l'appropriation et l'acculturation des TIC en comparant ces trois expériences. Sur les différents sites étudiés, la relation au temps constitue une dimension importante des apprentissages techniques dans la gestion de l'événement. Elle sera appréhendée ici à partir de trois types d'argumentations sur la temporalité, qui recoupent les trois étendues de la mémoire selon $\mathrm{M}$. Halbwachs : mémoire historique, mémoire collective et mémoire individuelle (Halbwachs, 1925). La première, objet de la première partie, prend donc racine dans le temps évolutionniste de l'histoire et montre concrètement comment les catastrophes sont de plus en plus instrumentées par les sciences et techniques, parmi lesquelles les technologies de l'information et de la communication. La seconde est au principe de la seconde partie. Elle se situe sur une échelle spatio-temporelle plus réduite et tient compte d'une mémoire collective reconstruite par les acteurs locaux pour qualifier techniquement l'événement et gérer l'incertitude au moyen des TIC. Enfin, dans une troisième partie, les récits biographiques 
et personnels sur les événements interviennent afin de donner progressivement sens à des usages plus citoyens des TIC dans la prise en charge de la catastrophe.

3 Les usages de la science et des technologies dans la gestion d'une catastrophe naturelle représentent des indices pour savoir si un risque sanitaire ou écologique est plus ou moins acceptable à l'échelle d'une collectivité. La société du risque suppose en effet, pour les groupes comme pour les individus, d'avoir intégré des contraintes environnementales et de pouvoir réagir face à ces contraintes grâce à des moyens technologiques sophistiqués. Les TIC sont là pour réduire l'incertitude - avions nous posé comme préalable à notre enquête de terrain -, car il n'existe pas de prévention ou de précaution comme suppression des risques mais comme limitation des conséquences, si bien que toute attitude qui n'envisagerait pas les effets de ses actions peut être jugée irresponsable au regard du bien commun que constitue la préservation de l'environnement, et son corollaire en termes de santé publique, le souci de soi. En matière de risques naturels, la réduction de l'incertitude s'évalue généralement aux nombres importants de controverses et d'études, de mesures de précaution et de réparation suite à différents incidents. Pour ce faire, on examine notamment la façon dont un problème environnemental monte en généralité dans l'opinion publique (Risques, 2001). Plus la rationalité scientifique et les pratiques technicistes deviennent opérantes dans le débat public, moins ses causes et son traitement s'avèrent incertains.

Sur les différents sites étudiés, la montée en généralité des événements n’a pas la même intensité, ni la même durée mais elle interfère sur les registres d'action. Cinq années séparent la première de la dernière catastrophe. Or, plus on avance vers la fin de la période étudiée, plus la crise est paramétrée et définie à l'aide de savoir-faire technologiques dès son commencement. Il est significatif de constater par exemple que l'on convoque spontanément en 2005 des arguments techniques permettant de désigner une procédure d'alerte défaillante. De la même façon, les dysfonctionnements des matériels et des hommes sont davantage invoqués pour expliquer la catastrophe. A Limoges, il faut attendre plusieurs semaines avant que se déploient des arguments, voire des controverses, mettant en jeu la responsabilité d'une institution ou d'un dispositif technologique dans la gestion de l'événement, telle la saturation du réseau téléphonique ou du serveur de messagerie d'EDF. A Abbeville, les débats d'experts autour de la montée des eaux s'engagent au bout de quelques jours. A Bourg-en-Bresse, il suffit seulement de quelques heures pour se rendre compte que l'étendue des dégâts est imputable à une cause technique, le problème de la régulation du débit de la rivière étant prioritairement invoqué. Certes, à Abbeville et à Bourg-en-Bresse, la constitution même de l'événement, des inondations, autorise davantage l'opinion publique à chercher d'emblée des explications rationnelles. En effet, l'eau des rivières et des nappes phréatiques est canalisée, régulée depuis toujours par l'homme. Mais on n'a jamais vu un simple mortel stopper les forces de la nature avec le vent, sauf dans la mythologie ou la science fiction. Le recours à l'expertise et à la technique est plus vif lorsque les causes des accidents ne peuvent pas être établies avec exactitude par la nature et la durée même de l'événement. Dans le cas des inondations à Abbeville et à Bourg-en-Bresse, on assiste à une "résidentialité proliférante» (Roux, 2001) du risque - sur plusieurs jours, voire sur plusieurs semaines - : les débordements sont "sournois", s'imposant notamment de manière inégale et irrégulière dans la Somme; ils sont attachés à un ou plusieurs quartiers de la ville mais les raisons de leur existence, comme dans le cas de la pollution industrielle des sols, sont invisibles et s'étendent bien au delà des murs. A Limoges, la 
catastrophe est paradoxalement rendue plus acceptable par sa violence, sa soudaineté et l'impuissance des hommes à arrêter les vents.

5 Comment les TIC participent concrètement à la maîtrise des plages d'incertitude concernant les catastrophes? En réalité, elles sont assez marginales si l'on considère seulement la mise sur agenda des catastrophes dans son contexte médiatique. Les usages numériques agissent de façon concurrente et/ou complémentaire avec d'autres vecteurs de communication: presse écrite, télévision, radio, radio-fréquence, téléphone filaire, bouche-à-oreille, etc. L'accès à ces ressources dépend bien évidemment des besoins exprimés par les différents acteurs à des moments distincts de la catastrophe et tient compte des spécificités techniques de chacune d'entre elles. De par son instantanéité, sa réactivité et sa fiabilité, la radio par exemple s'est révélée être un instrument très puissant dans le Limousin car l'isolement des personnes sinistrées était grand, surtout en campagne où le seul transistor à piles permettait de maintenir un lien avec le reste du monde.

6 A Bourg-en-Bresse, la presse écrite locale a constitué le principal support d'information et de délibération sur les inondations. Abbeville est restée dans les annales des catastrophes françaises comme étant le symbole d'une surmédiatisation, laissant place à des rumeurs et à des amplifications de l'événement les plus extravagantes. La ville aurait reçu plus de 80 télévisions nationales et internationales. Plus globalement, le poids des infrastructures de communication est à relativiser en situation de catastrophes naturelles parce qu'il se heurte à deux limites que les spécialistes des médias et les militaires connaissent bien lorsqu'ils se préoccupent, non des usages, mais de la transmission du signal de la communication. Il s'agit de la sécurisation et de la dilution de l'information. Lorsque la sécurisation se pose à un niveau technique, et bien qu'il soit toujours difficile de dissocier les infrastructures matérielles des mesures opérationnelles, les difficultés concernent en premier lieu la capture du signal. Ces difficultés sont de deux ordres : ou bien le canal physique de transmission (relais, batteries) est absent ou obsolète, ou bien les terminaux ou nœuds des réseaux (lignes, serveurs, routeurs) sont saturés. Sauf à œuvrer dans une proximité relationnelle très forte où le bouche-à-oreille est essentiel, ce qui est le cas à Abbeville et à Bourg-en-Bresse mais pas au niveau de l'agglomération de Limoges, l'absence de circuit de communication peut devenir un obstacle de premier ordre. C'est ainsi que dans le Limousin, toute la logistique s'est organisée d'emblée autour de la ré-alimentation en énergie dans la perspective initiale de sauver des vies. La sécurisation consiste encore à agir sur la confidentialité des messages pour éviter la panique ou pour réguler le flux d'information vers des organismes centralisateurs de la crise comme les préfectures. Les objectifs poursuivis vont de la protection des populations à la préservation d'une autonomie d'action vis-à-vis des médias. La dilution de l'information est souvent préjudiciable à la dynamique de concertation et au processus de décision. Le maire d'Abbeville nous apprend qu'il avait deux portables, le premier étant réservé à la gestion de crise dans sa ville, le second à ses relations avec la préfecture. Mais la multiplication des canaux de communication représente parfois une contrainte, notamment auprès des services d'urgence qui interviennent à grande échelle et qui disposent, chacun, en interne, de leur propre réseau numérique ou radioélectrique. Plus il y a d'intermédiaires dans la diffusion d'un message, plus celui-ci se disperse et prend du temps pour arriver à «bon port». Cela justifie que l'on se dote par exemple d'une unité de communication mobile et inter-opérable, où toutes les nouvelles 
puissent être entendues, intégrées et travaillées simultanément par différents services impliqués dans la gestion de crise.

7 Les limites fonctionnelles des TIC étant posées, il reste qu'elles sont de plus en plus présentes pour paramétrer les interventions selon les niveaux d'urgence avant, pendant et après les événements. Lors de la première étape, elles interviennent grâce à la mise en place d'outils de vigilance et d'alertes météo circulant notamment par messagerie électronique ou par téléphone portable (SMS). Ceux-ci sont disponibles mais encore très peu exploités sur nos trois terrains d'expérimentation, hormis dans les services d'intervention ou d'accompagnement de l'urgence (police, gendarmerie, pompiers, préfecture, Croix rouge, etc.). Malgré la volonté des services de l'Etat de généraliser un service d'alerte météo de type Vigimail, les technologies permettant de prévenir d'un danger imminent sont généralement peu invoquées dans les discours des personnes interviewées au cours de l'enquête ${ }^{1}$, et encore moins utilisés chez les habitants. Dès que le sinistre est déclaré, et dans la mesure où cela reste encore possible, les TIC sont convoquées pour faire des diagnostics, coordonner les acteurs de terrain et organiser l'aide aux populations. Leur utilisation vise à maximiser la localisation des problèmes (bases de données évolutives en temps réel par exemple) et la planification des actions sur le terrain (systèmes d'information territoriaux).

Ces usages sont au cœur des processus de décision et d'intervention, notamment au sein des services d'urgence, des cellules de crise dans les préfectures, les mairies et les services déconcentrés de l'Etat. Ils portent la marque de fonctions collaboratives et interactives sophistiquées, que l'on s'approprie là aussi différemment selon la nature, la durée mais aussi l'échelle de la catastrophe. A l'occasion de la tempête de 1999 à Limoges, les contraintes de communication étaient telles que la portabilité des terminaux d'informations a été jugée déterminante, ne serait-ce que pour déterminer les zones les plus exposées au danger. Dans le cas d'Abbeville, le téléphone mobile a suffi aux acteurs locaux pour créer un maillage opérationnel important.

Enfin, les TIC sont convoquées sous deux formes après les événements. Premièrement, elles représentent un moyen de contrôle pour les pouvoirs publics en cas de litige, grâce aux enregistrements des traces numériques préalablement effectués au cours de l'activité (appels téléphoniques, télé-surveillance, etc.). Ce nouveau besoin apparaît surtout lorsque la controverse est très vive sur la responsabilité de la catastrophe. A Bourg-en-Bresse, les habitants d'un grand immeuble dont les caves avaient été inondées, ont reproché aux pompiers de ne pas avoir actionné leur sirène, ce qui leur aurait permis de "sauver " leurs biens à temps. Ils se sont intéressés aussi aux dysfonctionnements d'une ou plusieurs vannes de régulation du débit de la rivière. Ces deux problèmes ont fait l'objet d'un litige impliquant directement les pompiers et le syndicat d'aménagement de l'affluent concerné. Dans les deux cas, les responsables des structures visées ont mentionné, pour les premiers, un enregistrement systématique des appels téléphoniques successifs qui ont paramétré l'alerte et, pour les seconds, une absence de défaillance du système de télésurveillance des vannes. Ce contrôle s'effectue alors à la minute, voire à la seconde près. Au delà de ces exemples, il pose le problème éminemment politique de savoir qui maitrise la traçabilité électronique dans un système d'information (Torny, 1999). Deuxièmement, les TIC favorisent l'argumentation et la délibération sur le risque naturel dans certaines conditions d'apprentissage. C'est sur ces conditions que s'attardera notre développement. 
10 Si l'on s'en tient toujours à un niveau rétrospectif, le recours aux TIC en situation de catastrophe naturelle a partie liée avec des compétences sociales qui ont été acquises grâce à des ressources disponibles dans la mémoire collective des acteurs. Celles-ci portent spécifiquement la marque d'un capital culturel et relationnel prédisposant à de meilleures appropriations technologiques dans la prise en charge de l'événement. Ainsi, l'engagement ou le concernement citoyen à la gestion de crise, indépendamment des traditionnelles mobilisations institutionnelles et associatives, est le fait d'individus qui ont une connaissance du risque dans sa physicalité et sa localité. Autrement dit, il s'agit de détenir une culture avisée du patrimoine naturel local et à pouvoir l'actualiser au moment opportun. La situation d'enquête a constitué en quelque sorte ce moment opportun, notamment grâce à la réactivation des souvenirs par les entretiens quelques années après les catastrophes de Limoges et d'Abbeville mais aussi grâce à la mise en place d'un forum de discussion sur le site dédié à l'étude http://www.technorisque.net, forum qui devait être opérationnel pour un événement non défini au démarrage et qui devint Bourg-en-Bresse en avril 2005. Ce sont, pour paraphraser J. Roux, des " passeurs en généralité " qui vont essayer de comprendre les faits en mettant en valeur des explications rationnelles sur les milieux où ils vivent. On assiste ainsi à une acculturation du danger où l'acceptabilité sociale de la catastrophe et la diminution de son caractère incertain se réalisent par une connaissance approfondie de sa genèse et de sa complexité. Dans le cas des inondations d'Abbeville et de Bourg-en-Bresse, on note une érudition impressionnante sur la nature des sols, sur la géographie de la rivière et les différents travaux qui ont modifié son cours ou son lit, sur le rôle historique des marais, des canaux ou des moulins, les dates et les circonstances précises des dernières inondations. Dans le cas de la tempête à Limoges, le parcours et l'intensité des vents, le relief de la région, son histoire arboricole et agricole ainsi que l'influence du relief sont bien investis.

11 L'étendue protéiforme du risque fait appel à des temporalités multiples dans l'argumentation. Ainsi, le « passeur en généralité » peut facilement faire le lien entre les différentes raisons qui ont conduit aux problèmes et il sait pertinemment qu'il n'y a pas d'interprétations linéaires, ni de responsabilités circonscrites de l'événement à une institution ou à un individu. Dans son discours, les différentes dimensions patrimoniales du risque naturel sont enchevêtrées et leurs combinatoires, même si l'intéressé n'en a pas forcément conscience, sont dépendantes d'un écosystème local. Le "passeur en généralité » semble donc avoir cette habileté cognitive qui est souvent mentionnée par les spécialistes de l'hypertexte dans l'appropriation durable des TIC, qui consiste notamment à pouvoir participer à plusieurs « micro-univers temporels » simultanément. Dans nos hypothèses de travail, nous avions établi un parallèle entre cette capacité cognitive et celle qui est souvent mobilisée dans les systèmes experts en environnement où il s'agit précisément d'apprécier une situation à risque dans sa pluridimentionalité, d'en cerner les contours et d'en proposer une définition compatible avec son traitement ultérieur (Dourlens, 1997).

La réflexivité sur la catastrophe signifie que toute conscience doit être prévenue de ce qui se passe "entre" les éléments d'un écosystème local. Elle renvoie à des modèles d'écologie cognitive, qui de la théorie de la complexité d'E. Morin à la critique de la décision de L. Sfez, permettent de gérer des rationalités juxtaposées travaillant de façon discontinue dans le temps et dans l'espace. De nombreux signes laissent à penser aujourd'hui que ce lien peut être fécond. En effet, ce sont souvent ceux qui maîtrisent le mieux les TIC qui peuvent plus facilement discerner l'horloge et les battements du risque 
naturel dans sa dimension historique. Deux exemples illustrent ce phénomène. Un responsable de service à Limoges, disposant d'une maitrise complexe et vaste de l'offre logiciel, allant des usages de l'Internet à la cartographie interactive, précise que dans un cas comme celui de la tempête, ce ne fut pas un événement, mais « 30000 événements » qui se sont produits simultanément à travers la ville. L'attention est mise ici sur une logique de situation qui prend en compte l'expérience des acteurs, qui agissent et sont agis par ce qui arrive le 27 décembre 1999. Cela sous-entend que le traitement de la catastrophe doit faire appel à des ressources locales multiples et préalablement identifiées, notamment grâce à des bases de données arboricoles à l'échelle de l'agglomération. A Bourg-en-Bresse, le forum de discussion a été utilisé par un certain nombre d'acteurs locaux. Les plus actifs sont ceux qui avaient une connaissance minimale du site inondé. L'un d'entre eux, originaire de la ville mais non du quartier touché par les inondations, a fourni à plusieurs reprises des informations sur la géographie des lieux, sur le taux de pluviométrie du mois d'avril 2005 en donnant les adresses URL de ses sources. Un autre, se référant explicitement à une mémoire de "vieux brugiens » ayant connu d'autres crues, se désole de constater que le boulevard situé près de la zone inondée avait coupé le cours du surplus centennal de la voie d'eau concernée par les débordements. On touche bien ici à la question de la connaissance rétrospective du risque pour comprendre et situer ce qui se faisait avant, lorsque le danger était inexistant ou lors des précédents sinistres. Cette compréhension se situe dans le champ d'expérience de "l'habiter", précise encore J. Roux (Roux, op. cit.). Elle se distingue d'une rationalité associative ou administrative mais s'ouvre à une communauté de vigilance fondée sur des échanges entre individus. Ici, le patrimoine constitué par l'écosystème local, suppose d'avoir intégré des contraintes environnementales et de pouvoir les mettre à disposition d'autrui. Toutefois, les organisations ne sont pas exsangues face à cette possibilité. La dimension patrimoniale et protéiforme du risque peut même devenir un enjeu de lutte et de pouvoir dont l'objet est de détenir tout ou partie de la mémoire locale. Les controverses d'Abbeville autour des vannages et autres canaux de la Somme, mettant en scène diverses instances chargées de les réguler, sont significatives de ce point de vue. Cette pratique de mémoire sociale est foncièrement attachée à l'histoire collective des lieux touchés par les catastrophes.

Les dispositions culturelles en faveur des usages numériques, à travers la dimension patrimoniale de la catastrophe, ont bien à voir avec l'inscription de la collectivité dans le temps, mais aussi dans l'espace. Or, ce dernier lien est encore plus évident avec ce que l'on a nommé précédemment "un capital relationnel». Les TIC, jusqu'à une période encore très récente, étaient encore vues de manière prophétique à travers le filtre d'un monde exclusivement virtuel où le temps réel et la déterritorialisation des échanges étaient sacralisés sur l'autel d'une ubiquité sans contrainte et sans limite. Nous avons tenté de travailler à l'inverse sur le postulat d'un ancrage géographique des compétences en faveur de la réduction de l'incertitude via les TIC, celles-ci pouvant s'appliquer notamment à la localisation des relations inter-subjectives entre les protagonistes de l'échange d'information. Ceux qui sont les plus enclins à utiliser des TIC en situation de catastrophes naturelles ont un parcours migratoire qui oscille entre une tendance à la mobilité, qui les a mis en contact avec des lieux hétérogènes en France comme à l'étranger, précédée et/ou suivie d'un enracinement très fort sur le territoire local. Cela ne veut pas dire que tous les « notables » des villes utilisent les TIC. 
14 Mais l'ancrage local reste un facteur favorisant l'assimilation de la technique sur la question des catastrophes. Nous retrouvons par ce biais la mise en valeur de la dimension patrimoniale et protéiforme du risque naturel, qui corrobore par ailleurs un certain nombre d'études socio-historiques sur les fondements culturels communautaires de l'écologie politique (Valarié, 1996). Ce sont principalement les acteurs de la contractualisation au niveau local, que l'on retrouve à Abbeville autour de cellules de crise particulièrement bien investies par les associations et à Limoges par un réseau de responsables politiques et administratifs très impliqués dans la gestion de crise. Les ressources de la localité vont jouer un rôle déterminant dans la capacité à entrer en communication inter-personnelle avec autrui. Cette communication s'enrichit prioritairement du face-à-face et de la poignée de main. Tous les protagonistes reconnaissent d'ailleurs que les différentes catastrophes ont fait naître de ce point de vue de véritables solidarités, quand ce ne sont pas des rencontres formelles au niveau professionnel. Un policier municipal à Abbeville admet qu'en deux mois continus de patrouilles en canoë puis à vélo, son équipe a gagné un an de travail dans l'îlotage et la connaissance des habitants. Un maire de l'agglomération de Limoges précise, quant à lui, que la catastrophe a permis de jeter les bases d'une communauté de communes avec le chef lieu de canton voisin. Cette communauté de commune s'est dotée depuis lors d'un Intranet. Il apparaît ici, dans la continuité d'ailleurs des travaux de M. Crozier et de B. Latour, que la stabilisation du maillage socio-technique relationnel autour des événements au niveau local contribue à réduire l'incertitude. Plus la force de l'accord obtenu ou attendu sur l'acceptabilité de la catastrophe repose sur un processus d'interconnaissance entre les acteurs, plus l'incertitude semble maîtrisée. Or, le niveau de l'action décentralisée, offrant plus de proximité physique et empirique entre les différents intervenants, est le maillon le plus approprié pour favoriser l'entraide et la participation. Les réseaux interpersonnels et les réseaux de proximité sont souvent la première source d'information des sinistrés, tout au moins au début du drame. Et les bénévoles actifs dans ces réseaux, comme à Abbeville, servent souvent de relais d'information, ce qui n'a pas été le cas à Limoges et à Bourg-en-Bresse, notamment parce qu'ils n'ont pas été impliqués dès le départ dans le processus de concertation.

L'on ne saurait pourtant se contenter d'une conception fixiste de l'espace pour expliquer l'importance des choix technologiques en situation de catastrophe naturelle. Certes, plus les densités relationnelles sont fortes, plus le passé des échanges constitue une ressource déterminante dans l'écheveau des décisions concernant l'événement, et par là même, dans l'usage qui est fait de l'innovation via les TIC (Alter, 2000). Mais ce constat ne s'arrête pas aux frontières communales ou intercommunales. Les dispositifs que nous avons analysés se constituent en réseaux, réseaux de réseaux, inter-réseaux, réseaux dans les réseaux, systèmes à géométrie variable qui convergent tous à un moment donné vers un objet commun: faire face à un risque naturel. Il s'agit d'une organisation dite «flexible» ou «souple» au sein de laquelle chaque personne ou chaque groupe peut se relier à tous pour faire cheminer et mettre en commun des savoirs avant, pendant et après les événements. Ainsi, l'acteur collectif s'organise selon des flux de circulation de connaissances mais ceux-ci ne sont pas dénués d'une assise géographique extraterritoriale, comme en témoignent les filiations politiques d'Abbeville avec le niveau décisionnel parisien par exemple. A l'inverse, une personne handicapée à Bourg-enBresse, habitant un immeuble dont l'ascenseur n'a pas fonctionné pendant 15 jours, se plaint sur le forum de n'avoir eu aucun recours auprès des officiels. Et de citer la régie de 
quartier, la mairie, le conseil syndical, la presse locale avant de conclure : Je n'ai « que» 58 ans et heureusement des amies.

Les TIC, dans ce contexte, ne contribuent pas à changer la nature des réseaux relationnels existants. Elles permettent de se déployer à partir de proximités affectives, sociales ou professionnelles. La contrainte géographique intervient lorsque le face-à-face est impossible, comme à Bourg-en-Bresse où l'on constate que ceux qui communiquent entre eux sur le forum pourraient très bien se rencontrer dans la rue ou au pied de leur immeuble en situation normale. Les TIC accentuent donc la mise en relation lorsque les rapprochements physiques sont difficiles, que ce soit dans l'espace du "proche » ou du « lointain ». A cet égard, mentionnons que l'âge avancé et l'isolement géographique se retrouvent être dans notre étude deux critères de vulnérabilité importants face aux aléas climatiques, surtout dans les campagnes et dans les quartiers périphériques des centres villes les plus touchés par les inondations, là où résident principalement des personnes âgées. Toutefois, plus la localisation des relations est étendue et reconnue dans sa spécificité organisationnelle et géographique, plus les moyens de communication, parmi lesquels les TIC, sont utilisés comme outils de travail et d'anticipation de l'événement, et non pas seulement comme outils de liaison.

17 Le «capital relationnel " peut même être une ressource mobilisable grâce aux TIC à travers les groupes de discussion ou des listes de diffusion permettant de réunir des communautés d'intérêts. Les TIC organisent ici des échanges civilisés à distance, loin de la spontanéité de la rencontre au niveau local. L'exercice de la civilité suppose de passer d'un espace de proximité à un espace ouvert, aux ramifications complexes où les liens inter-personnels reposent sur un exercice effectif de la mobilité. Cela n'est pas sans rappeler les analyses de M. Castells (Castells, 1998), pour qui les inégalités d'accès à l'espace public se polarisent aujourd'hui entre ceux qui ont accès au réseau et à la mobilité et ceux qui en sont privés. Les liens à distance sont reconnus aussi pour les effets qu'ils exercent, non sur la seule libération de la parole et des frustrations comme pour la fréquentation du forum de discussion par les habitants dans le cas des intempéries de Bourg-en-Bresse, mais sur la mise en synergie de compétences: réajustement de pratiques, mise en visibilité du réseau et de ses réussites, dysfonctionnements, échanges sur les échanges, savoirs sur les savoirs, prise de conscience de l'apprentissage et création de contiguïtés sociales pour un meilleur accès à une culture du risque.

Nous avons vu en quoi l'évolution historique des événements peut conduire à qualifier techniquement la catastrophe à partir de savoir-faire technologiques. Puis nous nous sommes attardés sur l'inscription sociale de la catastrophe dans la mémoire collective, qui amène certaines personnes particulièrement érudites sur le patrimoine local à détenir une position privilégiée dans la communication et d'autres - parfois les mêmes - à pouvoir organiser des échanges civilisés à distance. Nous allons maintenant aborder un autre aspect de la gestion numérisée de la catastrophe en nous attardant sur les conditions d'énonciation individuelles du risque. Il s'agit de voir comment s'effectue l'acculturation à la technique dans deux contextes discursifs particuliers : lorsque les acteurs agencent les différents discours sur les événements avec leur histoire de vie d'une part et lorsqu'ils sont amenés à débattre sur un forum de discussion dédié à une catastrophe particulière d'autre part. Dans les deux cas, nous accédons à des procédés argumentaires, qui, à partir des récits sur les événements et de leurs mises en scène, déterminent si un risque est plus ou moins acceptable au niveau personnel et plus ou moins négocié dans l'interaction inter-personnelle. 

voir comment agissent des appétences particulières en faveur des projets d'innovation et des TIC. Pour les personnes qui disposent d'un faible accès aux technologies, il apparait que le registre affectif et émotionnel sur les événements est prépondérant. Nous retrouvons ici l'importance des données factuelles sur la catastrophe, une tendance à la dramatisation, à la culpabilisation et des formules chocs qui expriment des tensions et des désarrois très forts au moment de l'alerte maximale. L'argumentation, dans ce cas, se focalise souvent sur une importante responsabilisation, voire une judiciarisation $\mathrm{du}$ risque, s'affirmant souvent aux dépens des collectivités territoriales et de l'Etat. Si l'on se raconte plus facilement en s'exposant personnellement, notamment sur le forum de discussion, c'est parce qu'il s'agit avant tout d'ancrer un temps psychologique dans la gestion d'un événement qui a interrompu brusquement le sens que l'on avait donné précédemment à son destin. Ici, la vocation des technologies reste essentiellement exutoire. A l'inverse, les personnes les mieux équipées et les plus aguerries aux TIC «se racontent " beaucoup moins et les réponses portant sur la dimension biographique des trajectoires d'apprentissage par exemple sont exprimées de façon laconique ou tout simplement éludées. Auprès d'elles, on ressent beaucoup moins la nécessité de tracer un fil continu entre le "vécu » et la catastrophe. Les événements qui ont eu lieu avant s'inscrivent facilement dans leur histoire selon une hiérarchie et une cohérence qui ne semble pas poser de problèmes particuliers. Ces événements peuvent même donner un sens projectif à la gestion des risques.

A Limoges, un ancien responsable des services d'information chez les pompiers déclare être revenu instinctivement au centre opérationnel avant la tempête : Pas d'alerte météo, suspicion d'événement! On savait qu'il y allait avoir quelque chose de grave. A chaque fois, c'est pareil. L'anticipation du risque est ici manifeste. Dans le même esprit, les professionnels qui ont un usage averti des TIC expriment une certaine aisance à pouvoir répondre de façon positive à la question de l'après-événement. L'expérience professionnelle antérieure justifie alors que l'on veuille bien prévoir l'avenir à l'aide des TIC. Dans tous les cas, plus l'acteur de la catastrophe parvient à traiter lui-même son histoire en invoquant de façon positive des chaînes causales indépendantes et préexistantes à l'événement luimême, que ce soit à travers son hérédité familiale ou professionnelle, plus son récit sera placé sous le signe d'une scénarisation du futur: La tempête a été vécue comme quelque chose de choquant, pas comme une catastrophe (...) Au contraire même, une certaine forme de réussite: les gens ont eu conscience de participer à quelque chose de grand, à une expérience dans laquelle ils se sont retrouvés. Dans ce cas de figure, les discontinuités et les ruptures dans les trajectoires n'ont pas ou peu d'importance si elles portent la marque d'une historicité, c'est-à-dire d'une capacité que possède l'individu ou la société à se produire elle-même en donnant sens à ses pratiques.

21 Cette disposition est favorisée par la possibilité d'aller et venir spontanément entre les différents éléments du passé, du présent et de l'avenir et doit être reliée aussi avec le fait de pouvoir se jouer des différentes flexibilités temporelles offertes par les outils technologiques. Les plus aguerris au plan technique ont un usage que l'on pourrait qualifier de polymorphe des TIC, c'est-à-dire qu'ils ont un maniement courant du portable, qu'ils naviguent aisément sur le Web en étant adroits pour faire des requêtes complexes et pour sérier ensuite les informations en fonction de leurs centres d'intérêts professionnels et personnels. La maitrise des logiciels de navigation, de messagerie et de bureautique va souvent de pair avec des usages plus pointus, allant du maniement de

Développement durable et territoires, Dossier 11 | 2008 
fonctions collaboratives à la manipulation de ressources documentaires éclectiques. L'accessibilité aux technologies repose aussi sur une importante asymétrie des pratiques de communication entre les " plus connectés » et les moins « connectés » : les premiers se servent en effet plus facilement des TIC pour se jouer de la synchronie ou de l'asynchronie des échanges dans la coordination des acteurs ou la réalisation d'une tâche. Plus les personnes interrogées ont une position hiérarchique dominante, plus elles utilisent leurs téléphones (portables ou fixes) pour maintenir constamment un lien avec leurs collaborateurs. Plus elles se gardent aussi la possibilité d'une communication différée selon l'interlocuteur en utilisant des filtres, tel le secrétariat ou le transfert d'appel. Elles pratiquent aussi volontiers «la décommunication", justifiant les choix de ne pas avoir d'ordinateur à la maison ou de ne pas s'en servir pour s'accorder des « respirations temporelles » selon le terme de F. Jauréguiberry (Jauréguiberry, 1998). Les moins « connectés » se sentent à l'inverse dominés par des outils qu'ils ne maitrisent pas ou trop peu. Ils ont la sensation de ne pas pouvoir s'abstraire de la communication. Il y a globalement un parallèle à faire entre le fait de s'approprier la technique comme son destin.

22 Le risque maîtrisé est d'autant plus recherché ou toléré que ce contrôle lui même est valorisé en tant que tel et affirme l'autonomie de celui qui l'exerce. L'auto-contrôle devient une référence symbolique. Au sein d'une société fortement individualisée, il est le corollaire d'une gestion de risque maîtrisée. Ainsi, la catastrophe deviendra plus acceptable lorsqu'elle est perçue comme évitable et lorsque les individus qui y sont exposés ont le sentiment de pouvoir s'y soustraire ou d'y faire face par eux-mêmes (Peretti-Wattel, 2000). Dans une certaine mesure, les TIC peuvent même aider à transformer l'infortune de la catastrophe en avantage personnel ou professionnel. L'engouement pour l'innovation se joue cette fois dans la capacité de la technologie à déterminer l'avenir de façon positive. Ainsi, pour ceux qui maîtrisent le mieux l'informatique (et non pas seulement la communication), la catastrophe relève davantage d'une «incertitude probabilisable» (Brunet et al., 2002): le risque peut être mesuré, construit et contré. Il fait l'objet d'un calcul rationnel grâce aux TIC.

23 L'examen approfondi des échanges interpersonnels sur le forum de discussion spécialement conçu pour les intempéries de Bourg-en-Bresse ${ }^{2}$ corrobore l'analyse précédente. Une majorité de participants, soit une dizaine environ, y prennent dans un premier temps un statut de victimes de la catastrophe. A l'inverse, ceux que ce l'on a appelé "les passeurs en généralité", au nombre de deux, se transforment en «médiateurs » et organisent une véritable réflexivité sur le risque. Leur rôle dans la communication, indépendamment du message patrimonial qu'ils véhiculent sur les inondations dans un des quartiers de la ville, consiste à organiser le cadre spatiotemporel de la rencontre virtuelle en faisant par exemple des liens URL vers l'extérieur de la ville ou du quartier. S'improvisant animateur, le «médiateur " est à l'écoute, il clarifie les points de vue et situe la place de chacun dans la discussion. Il montre également une relative neutralité, renvoie dos-à-dos les responsabilités de la catastrophe invoquées par les intervenants, fait le lien entre des points de vue antagonistes, interpelle sans dénoncer les partenaires présents ou absents de la discussion (modérateurs du site, experts, institutions, etc.). Il explicite et globalise encore les problèmes détectés, contribue à la relance des échanges et gère enfin les incompréhensions ou les conflits. Il accompagne ainsi la réflexion collective sur le risque, assimilable ici à un processus de «traduction », dirait B. Latour. L'activité d'apprentissage du collectif en ligne s'entend en 
effet à travers une capacité à décoder les enjeux portés par la complexité de la crise et à identifier des acteurs ou des éléments hétérogènes du sinistre en les mettant en relation les uns avec les autres. Car, repérer des savoirs sur la catastrophe, c'est les nommer, mais c'est aussi se nommer en tant qu'acteur légitime de l'interaction par des interrogations, des manques ou des recherches de renseignements. C'est encore les inscrire dans une dynamique intersubjective, un acteur collectif plus large, apprenant ou citoyen. Cela confirme qu'il existe, au sein du forum, une relation étroite entre distribution sociale de la connaissance et production d'une réduction de l'incertitude (Conein, 2004).

Ainsi, le débat, si réduit soit-il dans le cadre de cette expérimentation artisanale - nous ne sommes pas dans le cadre d'un dispositif technique spécialement conçu pour mettre en scène des controverses sur les risques telles que les conférences de citoyens par exemple -, s'est enrichi de procédés argumentatifs assimilables à ceux que Y. Barthe, M. Callon, et P. Lascoume préconisent dans le cadre d'une démocratie technique (Barthe et al., op. cit.). Pour répondre à une demande de vigilance accrue du citoyen sur les technosciences et à un besoin d'information sur la coordination des objets ou des hommes, ces auteurs envisagent une co-construction des savoirs entre experts et profanes afin d'agir dans un monde incertain. Près de 1200 connexions ont été enregistrées sur le site Technorisque dans les 15 jours qui ont suivi les intempéries de Bourg-en-Bresse, et près de la moitié se sont concentrées sur les seules pages du forum. Une trentaine de messages a été postée malgré une procédure d'inscription et de dialogue assez fastidieuse. Tandis que la presse écrite concentrait l'essentiel du débat public autour de la catastrophe avec des représentants d'associations et d'organisations qualifiées et repérées comme telles, le forum semble avoir jouer pleinement en faveur de la participation active d'une population invisible sur la scène locale. Totalement désinvesti par les autorités, cet espace de discussion est apparu comme une forme de réalisation cognitive et démocratique du risque naturel dans un cadre inter-subjectif. Cette expérience confirme l'intérêt croissant des pouvoirs publics et des citoyens pour les outils interactifs synchrones lors de catastrophes. Le cyclone Katrina, qui a balayé la Nouvelle Orléans en 2005, a d'ailleurs véritablement révélé ce phénomène avec un nombre impressionnant de blogs, de sites Internet et de forums spécialement créés après l'événement.

Nous avons montré que les TIC pouvaient être un phénomène d'apprentissage dans la survenue des catastrophes permettant de réduire l'incertitude face aux risques naturels. Lorsqu'elles représentent une simple technologie de diffusion de l'information, les outils numériques sont utilisés principalement pour paramétrer les alertes, établir des diagnostics et enregistrer les traces des activités. Il apparaitt alors que la sécurisation des infrastructures de communication, la réactivité et la publicité des technologies favorisent leurs accès. En revanche, leurs usages sont à relativiser dans le contexte d'une offre médiatique complémentaire ou concurrentielle, notamment avec la radio ou la télévision. Ils varient également selon la nature, la durée et l'échelle de la catastrophe. Lorsqu'elles sont associées à une technologie de la connaissance, un certain nombre de facteurs culturels favorisent leur appropriation: une connaissance du risque dans sa dimension patrimoniale et protéiforme, la présence d'un réseau de relations facilement mobilisable dans la gestion de crise et dans la communication, une interprétation positive des événements prédisposant à une meilleure assimilation de la technique d'une part et à une réflexivité face aux risques d'autre part. Enfin, lorsqu'elles sont envisagées comme un espace de civilité et de citoyenneté, il apparaît que des médiateurs, qui sont autant de "passeurs en généralité " sur le risque naturel sont également utiles à son 
accomplissement cognitif et démocratique. La possibilité de débattre sur la catastrophe avec des outils interactifs dédiés permet d'élaborer une socialisation de l'incertitude si les conditions de participation entre savoirs profanes et savoirs experts sont réunies à cet endroit. A cet égard, les TIC pourraient être attendues dans le giron des sciences humaines et sociales, là où la traçabilité de l'information n'est pas utilisée à des seules fins de contrôle et de surveillance mais dans une perspective réflexive et émancipatrice (Brossaud, Reber, 2007). Il échoit en effet toujours à l'expert de travailler sur les limites de toute technoscience qui consiste à produire du certain dans un contexte d'incertitude et de questionner cette tension entre une utopie rationalisatrice et sa dénégation, entre la quête de l'objectivité et la reconnaissance de ses difficultés.

\section{BIBLIOGRAPHIE}

Alter N, 2000, L'innovation ordinaire, Paris, PUF, 270 p.

Beck U., 2001, La société du risque. Sur la voie d'une autre modernité, Paris, Aubier.

Barthe Y., Callon M., Lascoumes P., 2001, Agir dans un monde incertain, Essai sur la démocratie technique, Paris, Seuil, 358 p.

Conein B., 2004, «Les communautés informatiques comme communautés épistémiques ", in Delamotte, E. (dir.), Du partage au marché. Regards croisés sur la circulation des savoirs, Lille, Le Septentrion, pp. 57-76.

Brossaud C. (Coord.), 2005, Production et circulation de quelques usages numériques en situation de catastrophes naturelles ", http://www.technorisque.net, Rapport de recherche, Ministère de l'Ecologie et du développement durable, GRASS-CNRS, novembre 2005, 84 p.

Brossaud C., Reber B. (dir.), 2007, Humanités numériques, 2 vol. « Nouvelles technologies cognitives et épistémologie ", " Socio-informatique et démocratie cognitive », Hermes Sciences Publications.

Brunet S., Bergmans A., Bertrand A. et al., 2002, L'expertise en questions : domestiquer l'incertitude dans la société du risque, PIE-Peter Lang, n³, 2002, 290 p.

Castells M., 1998, L’ère de l'information, Tome 1 « La société en réseau », Fayard (trad.).

Dourlens C. et Vidal-Naquet P., 1997, «L'expert tel qu'en lui-même », dans Ion J. et Peroni M., Engagement public et exposition de la personne, Ed. de l'Aube, Paris, pp. 35-45.

Ehrenberg A., 1995, L'individu incertain, Pluriel, Hachette.

Gilbert C. (dir.), 2003, Risques collectifs et situations de crise. Apports de la recherche en sciences humaines et sociales. Paris, L'Harmattan, $340 \mathrm{p}$.

Halbwachs M., 1994 (1925), Les cadres sociaux de la mémoire, Paris, Albin Michel.

Jauréguiberry F., 1998, « Télécommunication et généralisation de l'urgence », Sciences de la société $\mathrm{n}^{\circ} 44$, «Urgence et décision », pp. 83-96.

Latour B., 1999, Politiques de la nature, La Découverte, 383 p. 
Risques collectifs et situations de crise : bilan et perspectives, Session 1, Mécanismes de mise sur agenda des risques collectifs, CNRS, La Recherche, 9 février 2001, pp. 5-65 http:// www.technorisque.net/vuouvrages.php?id=12

Lascoumes P., 2001, La productivité sociale des controverses, Intervention au séminaire "Penser les sciences, les techniques et l'expertise aujourd'hui", 25 janvier 2001, http://histsciences.univparis1.fr/penserlessciences/semin/lascoume.html

Roux J., 2001, Histoire collective d'un risque inassignable : Le cas de la pollution industrielle des sols, dans Risques collectifs et situations de crise : bilan et perspectives, CNRS, La Recherche, 9 février 2001, pp. 43-54.

Torny D., 1999, « La traçabilité comme technique de gouvernement des hommes et des choses », Les cahiers de la sécurité intérieure, Risques et démocratie n³8, pp. 157-182.

Peretti-Watel P., 2000, « Pourquoi et pour qui un risque est-il acceptable ? Représentations du risque et inégalités sociales », Les cahiers de la sécurité intérieure n 38, pp. 9-33.

Valarié P., 1996, «Les politiques publiques d'environnement : de la science militante à l'écologie urbaine », dans La négociation des politiques contractuelles, L'Harmattan, 1996.

\section{NOTES}

1. Il s'agit principalement des acteurs qui sont intervenus pour gérer la catastrophe pendant les événements : représentants des services d'urgence, des administrations, du secteur associatif et habitants. Le lecteur pourra se référer au site http:// www.technorisque.net pour des détails sur la méthodologie empirique employée, il aura également accès aux différents rapports publiés et à une bibliographie commentée. 2. Pour informer les habitants de Bourg-en-Bresse de l'existence du forum sur le site Technorisque, notre action a consisté principalement à utiliser les médias locaux (radio, presse écrite) et à distribuer de petites affichettes dès le lendemain de la catastrophe. .Pour en savoir plus sur les attendus et les ressorts de cette démarche méthodologique, voir « Les technologies de l'information et de la communication, objet et outil d'une science en action : l'exemple du site technorisque.net » à l'adresse URL suivante : http:// w3.u-grenoble3.fr/publicisation/communications/brossaud.htm

\section{RÉSUMÉS}

Cet article montre comment des professionnels et des sinistrés qui ont été confrontés à des catastrophes naturelles sur trois territoires distincts - la tempête à Limoges en 1999, les inondations à Abbeville (2001) et à Bourg-en-Bresse (2005) - ont construit une histoire commune du risque au moyen des technologies de l'information et de la communication (TIC) : Internet, téléphone portable, bases de données, outils de travail partagés, etc. Les usages des TIC sont d'abord resitués concrètement avant, pendant et après les événements dans un contexte historique où les sciences et techniques sont de plus en plus sollicitées pour réduire les incertitudes liées aux menaces sanitaires et écologiques. On voit ensuite s'élaborer une culture 
du risque sur la base de compétences socio-cognitives et relationnelles particulières face aux événements et à leur prise en charge technologique. Nous examinons en dernier ressort le rôle des TIC dans l'apprentissage d'une argumentation et d'une délibération collective sur les catastrophes, notamment grâce à la mise en place d'outils dédiés à l'étude sur le site http:// www.technorisque.net.

This article shows how professionnals and disaster victims involved in natural catastrophes in three different areas - Limoges storm in 1999, Abbeville and Bourg-en-Bresse floods in 2001 and 2005 - built a commun risk story with Information and communication technologies (ICT) : Internet, mobiles, data bases, groupware, etc. At first, ICT uses are concretely approached before, during and after the events in a historic context where sciences and technology are growing up to reduce uncertainties of medical and ecological threats. Then, in the second part of this article, a risk culture is supported by socio-cognitive and relationnal competences towards events and their technological holding. At last, we examine ICT place in a collective argumentation and deliberation about the disasters, in particular with specific tolls on our net site corporate http:// www.technorisque.net.

\section{INDEX}

Mots-clés : catastrophes naturelles, culture du risque, démocratie technique, mémoire collective, technologies de l'information et de la communication (TIC)

Keywords : collective memory, information and communication technologies (ICT), natural disasters, risk culture, technical democracy

\section{AUTEUR}

\section{CLAIRE BROSSAUD}

Claire Brossaud est sociologue et chercheure contractuelle. Elle est spécialiste des usages numériques appliqués aux politiques urbaines, à l'environnement et aux méthodes en sciences sociales. Elle a travaillé notamment pour le Ministère de l'Environnement et du Développement Durable et le GRASS-CNRS. Elle a publié quatre ouvrages. claire.brossaud@gmail.com. 